\title{
An oscillating bubble device for direct measurement of molecular exchange processes at the air-liquid interface in the medium frequency range
}

\author{
J. Örtegren, K.-D. Wantke, and H. Motschmann ${ }^{\mathrm{a})}$ \\ Max-Planck Institute of Colloids and Interfaces, Am Mühlenberg 9, D-14476 Golm/Potsdam, Germany
}

(Received 9 May 2003; accepted 7 September 2003)

\begin{abstract}
An experiment for the investigation of the exchange dynamics of adsorbed and dissolved surfactants at the air-water interface is described. The experiment combines two established techniques: the method of the oscillating bubble generates in a well-defined fashion a nonequilibrium state by a periodic compression and expansion of the surface layer. The state of the adsorption layer is then investigated by surface second harmonic generation (SHG). SHG is a second order nonlinear optical technique, which probes only the adsorbed molecules. Contribution of the bulk is widely suppressed and information on the orientational order of the surfactant and the corresponding number density of the adsorbed species are gained. These data are of utmost importance to assess the established models of Gibbs adsorption layer. The combination of the nonlinear optical technique with a rapidly oscillating bubble imposes severe experimental hurdles such as the synchronization of the laser pulses with the state of the bubble or problems arising from a bad signal to noise ratio. The experimental setup discussed in this article overcomes these problems and outlines all relevant design parameters. () 2003 American Institute of Physics. [DOI: 10.1063/1.1623620]
\end{abstract}

\section{INTRODUCTION}

The rheological properties of fluid surfaces and interfaces are of importance for the stability of foams and emulsions, as well as for coating processes, or in biological processes involving transport or reaction of molecules at liquidliquid and liquid-air interfaces. The interfacial properties of fluids can be tuned by soluble surfactants. The first step in the characterization of a surfactant system is the measurement of the equilibrium surface tension isotherm which is then discussed within the framework of the Gibbs adsorption model. ${ }^{1,2}$ It states that the derivative of the surface tension isotherm yields the surface excess, while its second derivative gives the Gibbs elasticity. Even this first step bears some puzzle. Just below the critical micelle concentration (CMC) the surface tension isotherm usually drops in a nearly linear fashion with the logarithm of the bulk concentration. According to Gibbs this means that a significant decrease in the surface tension occurs at a more or less constant surface excess. This is a paradox and demands further measurements which provide direct structural information on the internal architecture of the interface which cannot be obtained by thermodynamic consideration.

The desired information can be gained by nonlinear optical techniques based on a second order effect such as such as second harmonic generation (SHG) and sum frequency generation $(\mathrm{SFG}){ }^{3-5}$ Second order nonlinear optical processes cannot be detected in media with inversion symmetry. Consequently, the bulk of a liquid does not contribute to the second order nonlinear optical signal at least within the

\footnotetext{
a) Author to whom correspondence should be addressed; electronic mail: hubert.motschmann@mpikg-golm-mpg.de
}

framework of the electric dipole approximation. At the surface, however, the inversion symmetry is broken, and generation of second harmonics is allowed. SHG or SFG probes selectively the topmost adsorption layer and is for this reason a powerful tool to complement the thermodynamic analysis. SHG has successfully been used to gain information about the surface concentration and the tilt angle of adsorbed surfactants or the underlying symmetry within the monolayer. ${ }^{4,6-13}$ Up til now, SHG or SFG has been mainly applied to study equilibrium properties but it is the dynamics which governs many phenomena and provides also the key for the understanding of the equilibrium properties of Gibbs adsorption layers.

The experimental investigation of the nonequilibrium state is demanding; the same holds for the theoretical description of the underlying processes. The interesting time range for classical soluble surfactant is the $1-100 \mathrm{~ms}$ time window since molecular exchange processes between adsorbed and dissolved species may occur in this time frame. A good overview about the various experimental approaches to enter the desired time regime is presented in the review article of Miller et al. ${ }^{14}$ The most successful approaches are experiments with bubbles which provide at least an estimate of the dynamic surface tension in the medium frequency range, $1 \mathrm{~Hz}-1 \mathrm{kHz}$. Some techniques are based on continuous formation of bubbles and measurement of either the shape of the bubble, or the pressure in the chamber. ${ }^{14-16}$ The major drawback of these experiments is the elimination of the influence of the bulk on the results. Moreover, the arrangement cannot be combined with optical reflection techniques as the position of the bubbles is not defined. Consequently all information about the surfactant distributions in these systems are based on indirect measurements and on 
particular models and their underlying assumptions.

Methods using oscillating bubbles provide a few advantages in this respect. ${ }^{17-23}$ The influence of the bulk can be accounted for by calibration measurements using water and decanoic acid. The precise knowledge of the area changes combined with the surface tension data gives direct access to surface dilatational elasticity and viscosity. Furthermore, the position of the bubble is well defined during oscillation, which opens up for the possibility to probe the surface by SHG. Such an experiment might bring light upon a few questions that have been raised as a consequence of the progress in this field of research in recent years. ${ }^{14-16}$ In particular, the question of the influence of the molecular exchange processes at fluid interfaces on surface rheology remains unanswered and is linked to the surfactant distribution in the interfacial layer.

At present, dynamic surface tension measurements on an oscillating bubble can be performed in the frequency range up to $500 \mathrm{~Hz}^{20-23}$ The dilatational modulus $\varepsilon(f, c i)$ can be retrieved from to the measurement. The dilatational modulus $\varepsilon(f, c i)$ is defined as

$$
\varepsilon(f, c i)=A(\Delta \gamma / \Delta A),
$$

where $\Delta \gamma$ represents the change in surface tension, $\Delta A / A$ is the relative change in surface area, $f$ 's the frequency, and $c i$ is the bulk concentration of surface active components. $\varepsilon(f, c i)$ is a complex frequency function which is dependent on both bulk diffusion and molecular exchange processes. Measured dilatational moduli demonstrate that the Lucassen/van den Tempel (LvdT) model ${ }^{24-26}$ correctly describes the mechanical behavior of surfaces of many surfactant solutions. At low frequencies the surfactant molecules diffuse between the bulk and the monolayer at the surface during the compression-dilation cycle, giving a low $\varepsilon(f, c i)$, which is increasing with frequency. At higher frequencies bulk diffusion is to slow to be of importance, and $\varepsilon(f, c i)$ reaches a plateau value which represents the experimental Gibbs elasticity $\varepsilon m$. The evaluation of the oscillating bubble experiments in the medium frequency range does, however, reveal a few discrepancies to the theoretical models. One of the puzzles is the much greater value of the Gibbs elasticity, $\varepsilon 0$, calculated on the basis of the surface tension isotherm equation, compared to the experimental high frequency limit of the surface elasticity, $\varepsilon m$, at concentration near the CMC. There is plenty of experimental evidence that this discrepancy is observed for many surfactant systems. ${ }^{21,23,24,27,28}$ The reason for the mismatch between high frequency limit of the surface elasticity and Gibbs elasticity may be due to the underlying assumptions about the structure of the adsorption layer in dynamic conditions. The effective surface area is generally not restricted to a monolayer, as assumed in the LvdT model. Again, the combination of the oscillating bubble with SHG provides the desired independent structural information.

In this article we discuss the first setup and measurements combining the oscillating bubble method with SHG. This technique allows for instantaneous measurement of the concentration of surfactants in the monolayer $\left(\Gamma_{m}\right)$ in the medium frequency range. Small modifications of the setup may also give access to information about the orientation of the surfactants in the same frequency range. The first measurements cover the frequency range $10-60 \mathrm{~Hz}$, but higher frequencies are obtainable by use of a smaller bubble.

\section{EXPERIMENTAL SETUP}

The oscillating bubble experiment generates a periodical nonequilibrium state. The adsorption layer is expanded and compressed in a well-defined fashion and the system response is monitored as a function of the frequency of the distortion. A device where the pressure transducer is used is extensively described in Refs. 20 and 21. The instrument allows a precise determination of the surface dilatational viscosity and elasticity in the mid frequency range (10-500 $\mathrm{Hz}$ ). In this work, a version of the oscillating bubble chamber was developed, which allows SHG measurements on a fast oscillating bubble. The aim is to retrieve the surface coverage of the amphiphile within the topmost adsorption layer as a function of the frequency. This information together with the elasticity data provides a more detailed picture on the molecular exchange process occurring at the interface and is of general interest for several scientific questions in the colloidal community. The desired experiment imposes several experimental challenges. Second harmonic generation requires the use of pulsed laser systems for the generation of sufficiently high field strength in order to observe nonlinear optical effects. This is commonly obtained by an active passive mode locked laser, which emits 20-30 ps pulses at a fixed repetition rate of $10 \mathrm{~Hz}$. Most commercial systems show a disturbing temporal jitter between the pulses. So the first challenge is the synchronization of the laser pulses with the state of the bubble since about 100-500 pulses are required to obtain a reasonable signal to noise ratio. The second challenge is to reliable pickup the second harmonic signal on an oscillating bubble. This has been achieved by focusing the fundamental on the tip of the bubble. The SHG signal scales with the square of the number of molecules. The smaller illuminated area leads to a significant trade-off in the intensity. This could be widely compensated by exploiting a total reflection geometry. In our setup the fundamental is incident from the solution. The third challenge is to maintain a defined bubble oscillation with a precise knowledge of the area changes. This is addressed by a special surface modification of the tip, which yields a sharp hydrophobic/hydrophilic boundary required for maintaining the three phases contact point.

A photograph of the oscillating bubble chamber is shown in Fig. 1, and a schematic drawing of the cross-sectional area of the chamber is depicted in Fig. 2. The chamber is filled with an aqueous solution of surfactants. At the tip of a glass capillary in the middle of the chamber, a small hemispherical bubble is formed. The capillary was silanized and broken before usage, leaving the capillary walls hydrophobic and the fracture area hydrophilic. A piezoelectric translator (LVPZT, P830.40, Physik Instrumente) is connected to the chamber, generating a sinusoidal deformation of the bubble volume, and consequently of the surface area and the bubble radius. The voltage over the piezoelectric translator is generated by 


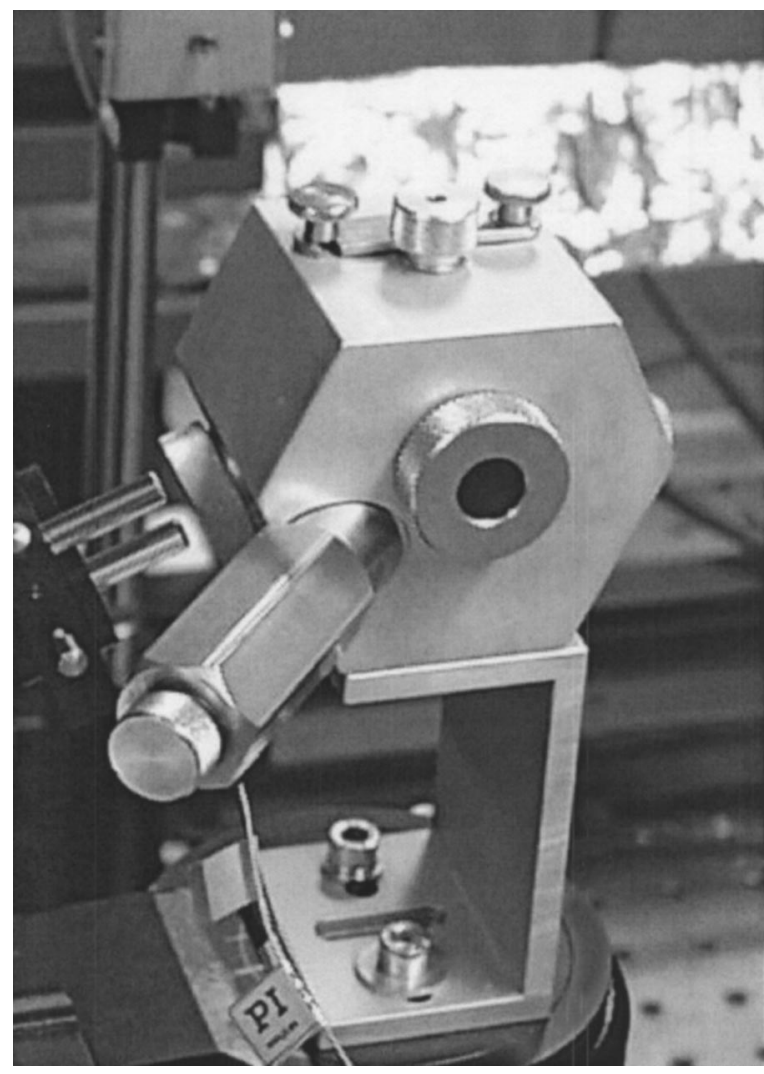

FIG. 1. Photograph of the chamber with the oscillating bubble.

a computer (A-WIN) and amplified (LVPZT-amplifier, model E-501.00, Physik Instrumente) before reaching the translator. The computer also triggers the laser, described later, and the trigger signal can be freely chosen during the sinusoidal voltage cycle. The fundamental light is coming into the chamber from the lower left part of Figs. 1 and 2, and is focused by a lens ( $F=16 \mathrm{~mm}, 063033$, Linos Photonics) onto the bubble. The lens is attached to a movable screw, making it possible to adjust the distance between the lens and the bubble. Light is incident in the total reflection mode at the bottom of the bubble at an angle of incidence of $68^{\circ}$. The frequency-

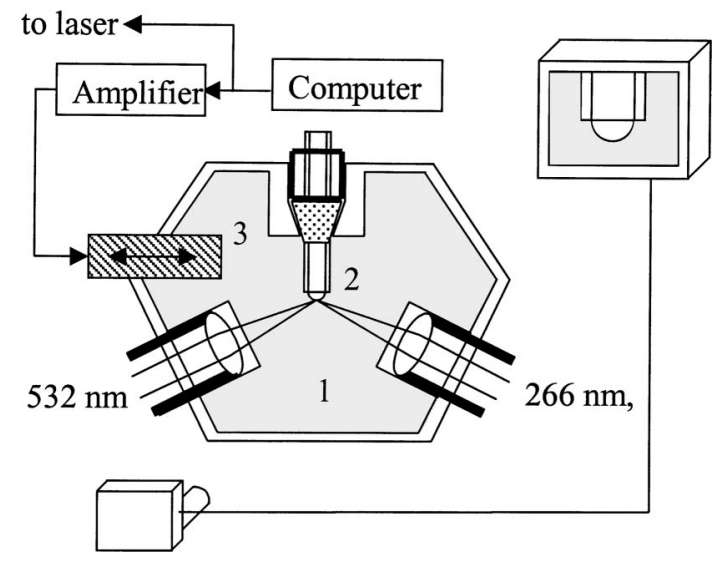

FIG. 2. The chamber with the oscillating bubble used in the SHG experiments. The fundamental light $(532 \mathrm{~nm})$ is focused by a lens onto the oscillating bubble at an angle where total reflection occurs. The fundamental and generated second harmonic light is collected by a quarz lens. (1) Surfactant solution (2) capillary, and (3) piezoelectric translator. doubled light generated at the bottom of the bubble is collected by a quarz-lens $(F=20 \mathrm{~mm}, 063316000$, Linos Photonics) attached to an adjustable screw at the rear end of the chamber, and exiting in the lower right part of Figs. 1 and 2.

The SHG measurements were carried out with the frequency-doubled light at $532 \mathrm{~nm}$ of an active/passive mode-locked Nd:yttritium-aluminum-garnet (YAG)-laser (B.M. Industries YAG 502 DPS 7910DP). The laser pulses were $35 \mathrm{ps}$, and the repetition rate $10 \mathrm{~Hz}$. The experiment is fully controlled by a computer which synchronizes the laser pulses with the bubble oscillation accounting also for the temporal jitter between subsequent pulses, a common feature of flash lamp pumped active passive mode locked Nd:YAG laser systems. The setup guarantees that the laser pulse hits the bubble at a user defined phase within the sinusoidal bubble oscillation. In this way $100-500$ pulses can be recorded under exactly identical conditions which is required to obtain a reliable signal to noise ratio. A common problem under Windows environment is an extremely poor real time capability. For this reason we use a Sharc ADSP 21062 processor for the all time critical processes and reduced the function of the Windows environment for the data visualization. The Sharc ADSP processor triggers the laser and generates simultaneously an oscillating output voltage for the piezotranslator at the desired frequency. Upon receiving the trigger signal, the flashlamps of the laser are charged. At the end of the charging process, the laser sends a TTL trigger signal back to the Sharc processor. The laser pulse is then emitted exactly $250 \mu$ s after this trigger which is sufficient to fine correct the phase of the bubble oscillation as our electronics react to the trigger within less than $0.1 \mu$ s and maintain a defined phase relation between oscillation and subsequent pulses.

The laser light was plane-polarized before the surfactant solution using a Glan-Thomson prism (extinction ratio 10-6, type $K$, Steeg \& Reuter). The plane of polarization was rotated $39^{\circ}$ from vertical polarization, where SHG is independent of the distribution of the molecular orientation. ${ }^{11}$ Spectral purity of the incoming light was assured by use of an infrared cutoff filter (BG39, Schott) in conjunction with a narrow-band interference filter (532 BP, LOT-Oriel). The frequency-doubled light generated at the interface was separated from the fundamental light by use of a visible cutoff filter (UG5, Schott) and a narrow-band interference filter (266 BP5, LOT-Oriel). The SH light was detected by a photomultiplier tube (R1398, Hamamatsu) with a quantum efficiency of $15 \%$, and a cathode radiant sensitivity of $35 \mathrm{~mA}$ $\mathrm{W}-1$ at $266 \mathrm{~nm}$. The supply voltage over the photomultiplier tube was $\sim 1850 \mathrm{~V}$ (V5D, Seefelder Messtechnik). The detected signal was processed by an oscilloscope (54720D, Hewlett-Packard) and integrated by a computer program.

The amplitude of the sinusoidal voltage over the piezoelectric translator was $30 \mathrm{~V}$, giving a relative amplitude $(\Delta A / A)$ of the area of the bubble during oscillation of 0.18 \pm 0.04 , as studied by optical means. We define the phase angle $\Psi$ of the bubble during oscillation as $90^{\circ}$, and $270^{\circ}$, when the bubble obtains its minimum and maximum volume, respectively. Consequently, at the phase angles $0^{\circ}$ and $180^{\circ}$, the bubble is at its equilibrium volume. In measuring SHG at 


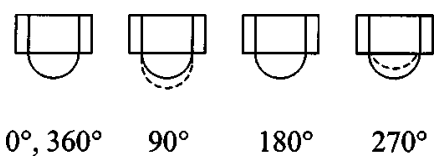

FIG. 3. Definition of the phase angle $\psi$ during bubble oscillation. The dotted curve is the volume of the bubble in equilibrium, and the full curve is the volume of the bubble as it is hit by light.

different phase angles, two obstacles must be overcome: First: the detected SHG signal is proportional to the square of the intensity of the fundamental light. This means that as the bubble oscillates, a small spatial deviation from focus at various phase angles may cause a change in the detected SHG signal. Second: the curved surface in combination with a spatially limited area of the lens collecting the outcoming light leads to a loss of some of the SHG intensity. Therefore, the shape of the bubble at minimum volume $\left(\Psi=90^{\circ}\right)$, having a less curved surface, may lead to a higher SHG signal. These problems were solved by adjusting the equilibrium volume of the bubble in the following way: in the case of measuring at a phase angle of $90^{\circ}$, the equilibrium volume of the bubble was made slightly larger than a half-sphere, so that the bubble was exactly a half-sphere as it was hit by light. Analogically, in the case of measuring at a phase angle of $270^{\circ}$, the equilibrium volume of the bubble was made slightly smaller than a half-sphere, again so that the bubble was a half-sphere as it was hit by light. In the case of the phase angles $0^{\circ}$ and $180^{\circ}$ the bubble was a half-sphere both in equilibrium and as it was hit by light. Consequently, light hit the bubble at exactly the same position and same size at all $\widetilde{\Psi}$ The definition of $\Psi$ is depicted in Fig. 3 .

The shape and size of the oscillating bubble in the SHG setup was studied by optical means through the small window shown in the middle of Fig. 1. The image of the bubble was detected by a charge coupled device camera (EHDkamPro02, EHD Imaging $\mathrm{GmbH}$ ) and transferred to a screen. A sharp image of the bubble during oscillation was obtained by using the picosecond laser pulses as only light source. According to these observations, the shape of the bubble was hemispherical up to $60 \mathrm{~Hz}$. The measurements presented in this article were performed at a bubble oscillation frequency of $40 \mathrm{~Hz}$. Figures 4(a) and 4(b) show photographs of the bubble at $40 \mathrm{~Hz}$, and at phase angles $\Psi=90^{\circ}$ and $\Psi$ $=270^{\circ}$, respectively. For frequencies above $60 \mathrm{~Hz}$ the shape of the bubble deviates a little from the spherical geometry due to the oscillations. Therefore, the reflection is somewhat different, and the comparison of the results is uncertain. The diameter of the bubble in this first series of measurements was $2 \mathrm{~mm}$. By decrease of the bubble diameter, an extended frequency range of 3-500 $\mathrm{Hz}$ can be reached as have been demonstrated by dynamic surface tension measurements in a bubble arrangement. ${ }^{20-23}$

An aqueous solution of the fluortenside 6-((2-hydroxyethyl)-\{2-[4-(4-trifluoromethyl-phenylazo)-phenoxy]-ethyl\}amino)-hexane-1,2,3,4,5-pentaol (F381) was prepared. This fluorchemical-based surfactant was prepared by Prescher and filter purified by recrystallization. Water was purified with a Milli- $Q$ system. The CMC of F381 in water is $\sim 200 \mu \mathrm{M}$. The ultraviolet (UV) spectrum was measured in an Agilent

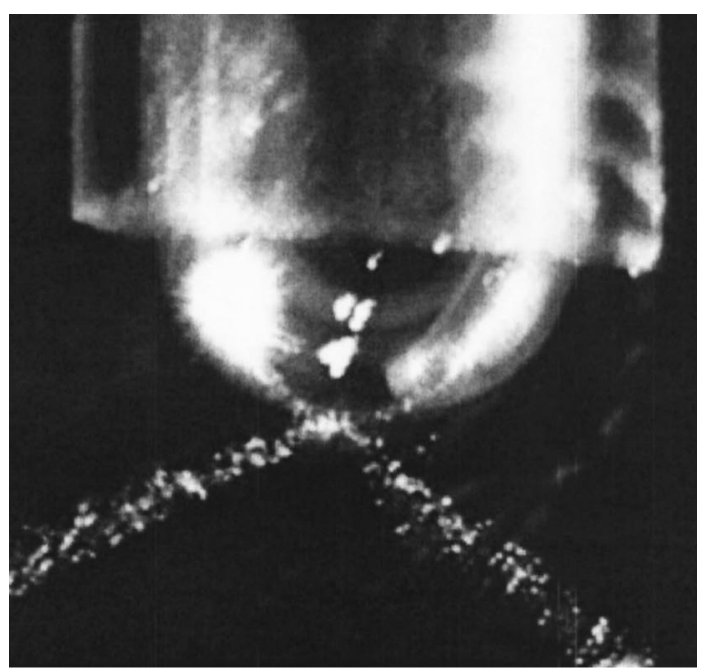

(a)

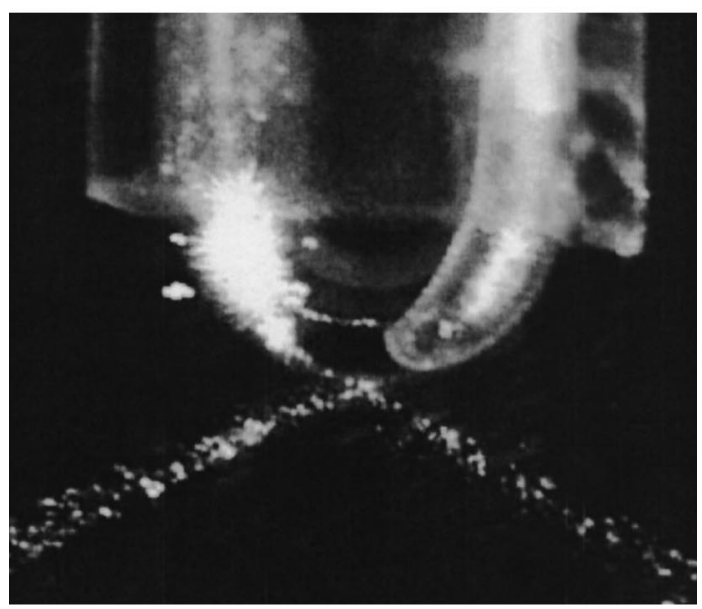

(b)

FIG. 4. Photograph of the oscillating bubble at an oscillation frequency of $40 \mathrm{~Hz}$, with a picosecond laser as only light source. (a) Phase angle $\psi$ $=90^{\circ}$ and (b) phase angle $\psi=270^{\circ}$.

8453 UV-visible spectrophotometer and reveals that the resonance frequency is positioned at about $340 \mathrm{~nm}$, and the solution is transparent at $532 \mathrm{~nm}$, and exhibits a dip in absorption close to $266 \mathrm{~nm}$. These features make the system well suited for SHG measurements in the oscillating bubble configuration where the fundamental light has a wavelength of $532 \mathrm{~nm}$. SHG measurements at the flat air-liquid interface of a surfactant with the same SHG-active group as F381 have been reported in the literature. ${ }^{9,13}$ The hyperpolarizability of the chromophore is completely dominated by its $\beta_{z z z}$ component, with $z$ being along the chromophore long axis. Therefore, the other $\beta$ components can be neglected, and the $\beta$ tensor can be treated as a scalar quantity. This simplifies the analysis a great deal. Under certain simplifying assumptions, the oriented gas model provides a relation between the effective second-order susceptibility $\left[\chi_{\mathrm{eff}}^{(2)}\right]$ and $\beta$ according to

$$
\chi_{\text {eff }}^{(2)} \propto \sum_{\substack{\text { molecules } \\ \text { at surface }}} \beta \propto \Gamma_{m}\langle\beta\rangle,
$$




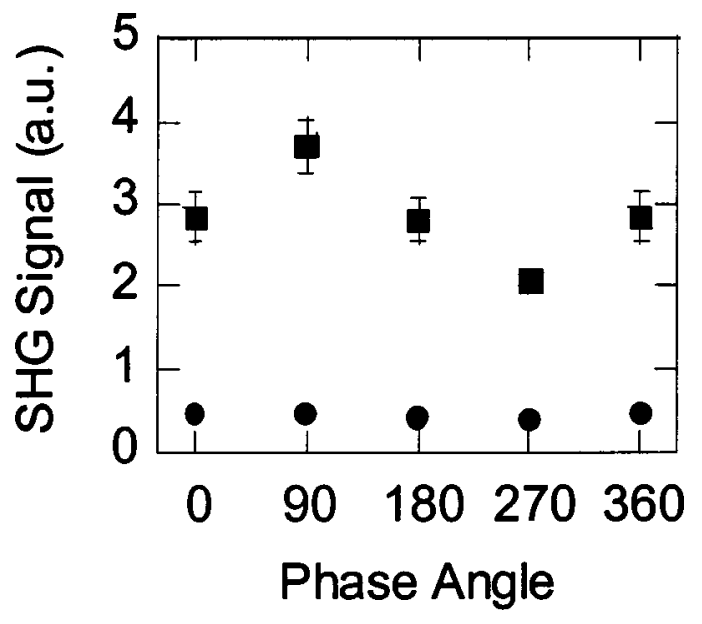

FIG. 5. SH intensity reflected by the oscillating bubble, as a function of phase angle $\psi$. Bubble oscillation frequency: $40 \mathrm{~Hz}$. (ロ) $30 \mu \mathrm{M} \mathrm{F} 381$ in water and $(\mathbf{)}$ water.

where $\Gamma m$ is the concentration of $\beta$-active units in the monolayer, and $\langle\beta\rangle$ is the spatial and orientational average of the second order polarizability. The reflected or transmitted second harmonic light intensity $(I 2 \omega)$, being generated at an interface, scales to $\chi_{\mathrm{eff}}^{(2)}$ and the incoming light intensity $(I \omega)$ in the following way:

$$
I^{2 \omega} \propto\left[\chi_{\text {eff }}^{(2)}\right]^{2}\left(I^{\omega}\right)^{2} .
$$

By the combination of Eqs. (2) and (3), the relative monolayer concentration, $\Gamma m$, is obtainable by measurement of SHG light intensity, $I 2 \omega$. At least ten measurements were performed at each phase angle, where each measurement is the average of the intensity of 100-200 laser pulses of SH light reflected at the bubble surface.

The capability of the setup is demonstrated in Fig. 5, where SHG measurements on an oscillating bubble in a 30 $\mu \mathrm{M}$ aqueous solution are shown together with SHG measurements on an oscillating bubble in pure water. The oscillation frequency was $40 \mathrm{~Hz}$, and the outcoming SHG intensity was detected at four phase angles. The SHG signal from the bubble in the surfactant solution is phase angle dependent. The highest SHG signal is detected at maximum compression $\left(\Psi=90^{\circ}\right)$, and the lowest at maximum dilation $\left(\Psi=270^{\circ}\right)$. The SHG signal from water is clearly smaller and does not show a phase angle dependence. This is a strong indication of that the outcoming SHG signal is generated by the surfactant molecules adsorbed at the surface.

The SHG data in Fig. 5 will now be evaluated and compared with the measured dilatational modulus $\varepsilon(f, c i)$ and the LvdT model. Dynamic surface elasticity measurements show that the surfactant system is purely surface elastic in the frequency range $10-60 \mathrm{~Hz}$ (i.e., $\varepsilon$ is constant) and that the system is well described by the LvdT model. ${ }^{29}$ The LvdT model predicts that the number of surfactants in the monolayer $(\mathrm{Nm})$ is constant at frequencies where bulk diffusion does not occur (here: above $5-10 \mathrm{~Hz}$ ). Then, the change in the concentration of surfactants in the monolayer $(\Delta \Gamma m)$ during oscillation is obtained by differentiating $\Gamma m$ $(=N m / A)$ at constant $\mathrm{Nm}$, giving

$$
\frac{\Delta \Gamma_{m}}{\Gamma_{m}}=-\frac{\Delta A}{A} .
$$

From the SHG data in Fig. 5, $\Delta \Gamma m / \Gamma m$ may be calculated by use of Eqs. (2) and (3). The result is 0.17 , which is, within experimental accuracy, equal to the relative change in bubble area $(-\Delta A / A=0.18)$. This means that Eq. (4) is fulfilled, and that the SHG data presented in Fig. 5 are in accordance with the dynamic elasticity measurements and the LvdT model. Moreover, SHG measurements performed in the frequency range $10-60 \mathrm{~Hz}$ gave the same behavior and absolute values as the data presented in Fig. 5. To conclude, we were able to directly measure the change in the concentration of surfactants in the monolayer at oscillation frequencies between 10 and $60 \mathrm{~Hz}$. The presented data confirms the predictions made from dynamic elasticity measurements and by the LvdT model.

\section{ACKNOWLEDGMENTS}

The authors highly appreciate stimulating discussions with Professor Möhwald, MPI-KG as well as the his continuous support. Furthermore the financial support from the foundation Blanceflor Boncompagni-Ludovisi, born Bildt, the Max-Planck Society and the DFG are gratefully acknowledged.

${ }^{1}$ J. Stauff, Kolloidchemie (Springer, Berlin, 1960).

${ }^{2}$ A. W. Adamson and A. P. Gast, Physical Chemistry of Surfaces (Wiley, New York, 1997).

${ }^{3}$ Y. R. Shen, The Principles of Nonlinear Optics, (Wiley, New York, 1984).

${ }^{4}$ K. B. Eisenthal, Chem. Rev. (Washington, D.C.) 96, 1343 (1996).

${ }^{5}$ G. L. Richmond, Chem. Rev. (Washington, D.C.) 102, 2693 (2002).

${ }^{6}$ V. Vogel, C. Mullin, and Y. R. Shen, Langmuir 7, 1222 (1991).

${ }^{7}$ S. Lehmann, G. Busse, M. Kahlweit, R. Stolle, F. Simon, and G. Marowsky, Langmuir 11, 1174 (1995).

${ }^{8}$ K. Kajikawa et al., Langmuir 12, 580 (1996).

${ }^{9}$ S. Bae, M. Harke, A. Goebel, K. Lunkenheimer, H. Motschmann, and D. Prescher, Langmuir 13, 6274 (1997).

${ }^{10}$ Y. Kinoshita, B. Park, H. Takezoe, T. Niori, and J. Watanabe, Langmuir 14, 6256 (1998).

${ }^{11}$ G. J. Simpson and K. L. Rowlen, J. Am. Chem. Soc. 121, 2635 (1999).

${ }^{12}$ M. Oh-e, S.-C. Hong, and Y. R. Shen, J. Phys. Chem. B 104, 7455 (2000).

${ }^{13} \mathrm{H}$. Motschmann and K. Lunkenheimer, J. Colloid Interface Sci. 248, 462 (2002).

${ }^{14}$ R. Miller, V. B. Fainerman, A. V. Makievski, M. Ferrari, and G. Loglio, in Handbook of Applied Surface and Colloid Chemistry, edited by K. Holmberg (Wiley, West Sussex, 2002), Vol. 2, Chap. 12.

${ }^{15}$ S. S. Dukhin, G. Kretschmar, and R. Miller, Dynamics of Adsorption at Liquid Interfaces, Studies in Interface Science Series Vol. 1 (Elsevier, Amsterdam, 1995).

${ }^{16}$ Drops and Bubbles in Interfacial Research, Studies in Interface Science Series Vol. 6, edited by D. Möbius and R. Miller (Elsevier, Amsterdam, 1998).

${ }^{17}$ G. Kretzschmar and K. Lunkenheimer, Ber. Bunsenges. Phys. Chem. 74, 107 (1970).

${ }^{18}$ K.-D. Wantke, R. Miller, and K. Lunkenheimer, Z. Phys. Chem. (Leipzig) 261, 1177 (1980).

${ }^{19}$ K.-D. Wantke, K. Lunkenheimer, and C. Hempt, J. Colloid Interface Sci. 159, 28 (1993).

${ }^{20}$ H. Fruhner and K.-D. Wantke, Colloids Surf., A 114, 53 (1996).

${ }^{21}$ K.-D. Wantke, H. Fruhner, J. Fang, and K. Lunkenheimer, J. Colloid Interface Sci. 208, 34 (1998).

${ }^{22}$ H. Fruhner, K.-D. Wantke, and K. Lunkenheimer, Colloids Surf., A 162, 193 (1999).

${ }^{23}$ K.-D. Wantke and H. Fruhner, J. Colloid Interface Sci. 237, 185 (2001). 
${ }^{24}$ J. Lucassen and R. S. Hansen, J. Colloid Interface Sci. 23, 319 (1967).

${ }^{25}$ J. Lucassen and M. Van den Tempel, J. Colloid Interface Sci. 41, 491 (1972).

${ }^{26}$ J. Lucassen and M. Van den Tempel, Chem. Eng. Sci. 27, 1283 (1972).
${ }^{27}$ Q. Jiang, Y. Chiew, and J. E. Valentini, Langmuir 8, 2747 (1992).

${ }^{28}$ Q. Jiang, J. E. Valentini, and Y. Chiew, J. Colloid Interface Sci. 174, 268 (1995).

${ }^{29}$ (unpublished). 